\title{
Existence of urban-scale macroscopic fundamental diagrams: Some experimental findings
}

\author{
Nikolas Geroliminis *, Carlos F. Daganzo \\ Institute of Transportation Studies and Department of Civil and Environmental Engineering, University of California, \\ 416E McLaughlin Hall, Berkeley, CA 94720, USA
}

Received 10 October 2007; received in revised form 8 February 2008; accepted 8 February 2008

\begin{abstract}
A field experiment in Yokohama (Japan) reveals that a macroscopic fundamental diagram (MFD) linking space-mean flow, density and speed exists on a large urban area. The experiment used a combination of fixed detectors and floating vehicle probes as sensors. It was observed that when the somewhat chaotic scatter-plots of speed vs. density from individual fixed detectors were aggregated the scatter nearly disappeared and points grouped neatly along a smoothly declining curve. This evidence suggests, but does not prove, that an MFD exists for the complete network because the fixed detectors only measure conditions in their proximity, which may not represent the whole network. Therefore, the analysis was enriched with data from GPS-equipped taxis, which covered the entire network. The new data were filtered to ensure that only full-taxi trips (i.e., representative of automobile trips) were retained in the sample. The space-mean speeds and densities at different times-of-day were then estimated for the whole study area using relevant parts of the detector and taxi data sets. These estimates were still found to lie close to a smoothly declining curve with deviations smaller than those of individual links - and entirely explained by experimental error. The analysis also revealed a fixed relation between the space-mean flows on the whole network, which are easy to estimate given the existence of an MFD, and the trip completion rates, which dynamically measure accessibility.
\end{abstract}

(c) 2008 Elsevier Ltd. All rights reserved.

Keywords: Urban mobility; Traffic congestion; Macroscopic fundamental diagram

\section{Introduction}

Various theories have been proposed for the past 40 years to describe vehicular traffic movement in cities on an aggregate level. These works have attempted to predict both the average and the distribution of speed in an urban area as a function of explanatory variables that characterize the demand and the network infrastructure.

\footnotetext{
* Corresponding author. Tel.: +1 510643 2310; fax: +1 5106421246.

E-mail addresses: nikolas@berkeley.edu (N. Geroliminis), daganzo@ce.berkeley.edu (C.F. Daganzo).
} 
Smeed (1966) theorized based on dimensional analysis that the maximum flow that can enter the central area of a city should be a function of the area of the city, the fraction devoted to roads and the capacity of the roads, expressed in vehicles per unit time per unit width of road. Although seminal, this work does not say what happens to speeds and trip completion rates when demand exceeds capacity during a rush hour.

Thomson (1967) found from data collected from streets in central London for many years that there seemed to be a linear-decreasing relationship between average speed and flow. ${ }^{1}$ Shortly thereafter, Wardrop (1968) proposed a generic relation between average speed and flow, which depended on average street width and average intersection spacing, but it still decreased monotonically. Zahavi (1972) analyzed relations for various cities in United Kingdom and United States by combining data across different regions of a city for the same time period (one day or peak period), and proposed that speed was inversely related to flow; i.e., still monotonically. Monotonicity only makes sense if traffic is light, since it cannot capture crowded states with very low speeds and flows; e.g., approaching gridlock. Thus, these models cannot be used to describe the rush hour in a congested city.

A related theory is the two-fluid model in Herman and Prigogine (1979). It asserts that the average speed in an urban area is a function of the fraction of vehicles that are stopped at any given time. Herman and Ardekani (1984) tested the theory and further proposed that the fraction of stopped vehicles was a power function of the density. This proposal allowed for a more realistic representation of crowded conditions in the steady state, but the idea was not sufficiently developed to create a macroscopic model with variable inputs and outputs that could describe a rush hour dynamically.

This step was taken in Daganzo $(2005,2007)$ with a framework that related the rate at which vehicles leave a network (the trip completion rate) to the number of vehicles in the network (the accumulation). These references argued that the relationship should hold in the time-dependent case for homogeneously congested regions of cities that were called "neighborhoods", if external conditions (e.g., the demand) change slowly with time. With accumulation as a state variable, these paper described the dynamics of the rush hour, the gridlock phenomenon, and how to improve accessibility by managing accumulation. Geroliminis and Daganzo (2007) later recast this theory in terms of two postulates: (i) that homogeneously congested "neighborhoods" exhibit an MFD relating "production" (the product of average flow and network length) and "accumulation" (the product of density and network length) and (ii) that the trip completion rate is proportional to the production. This reference demonstrated with numerous micro-simulations of the rush hour in downtown San Francisco (with very different demand distributions both in time and space) that the MFD indeed exists independently of the demand, that accumulation can be dynamically predicted, and that perimeter control schemes for improving accessibility work as expected.

These results were encouraging. They suggest that, conditional on accumulation large networks behave predictably and independently of their origin-destination tables. If the results hold up to further scrutiny, practitioners will have reliable tools to both, anticipate the results of "smart" traffic management policies, and plan accordingly without the uncertainty inherent in today's forecast-based approaches. This further scrutiny should include field experiments because simulations invariably include untested assumptions.

As an attempt in this direction, this paper analyzes data from a natural experiment that took place in Yokohama (Japan). Section 2 describes the site and the raw data, which came both from fixed detectors and taxis. Section 3 shows from the fixed detector data that an MFD exists for the part of the Yokohama network that is covered by detectors. Section 4 then demonstrates, by fusing taxi and detector data, that a city-wide MFD likely exists; this section also shows that the important trip completion rate can be predicted from observable data. Finally, Section 5 discusses the results and suggests directions for further work.

\section{Site and data description}

Yokohama is a major commercial hub of the Greater Tokyo Area. It developed rapidly as Japan's prominent port city with a population of 3.6 million. Its road network includes streets of various types, with closely spaced signalized intersections at its center $(100-300 \mathrm{~m})$, and a few elevated freeways. Streets have 2-4 lanes in each direction. The speed limit is $50 \mathrm{~km} / \mathrm{h}$ on arterials. Major intersections are centrally controlled by

\footnotetext{
${ }^{1}$ This suggests to us that the streets in the data set were not very congested.
} 
multiphase traffic signals with a cycle time that responds to traffic conditions: $110-120 \mathrm{~s}$ long at night and 130$140 \mathrm{~s}$ during the day. The part of downtown Yokohama examined in this paper is approximately a $10 \mathrm{~km}^{2}$ triangle with corners at Yokohama Station, Motomachi-Chukagai Station and the Shin-Hodogaya Interchange. The center of this region is congested during the weekday's peaks, with average speeds dipping below $10 \mathrm{~km} / \mathrm{h}$. Data from two different sources, and spanning one month (December 2001), were available:

- Fixed sensors: 500 ultrasonic and loop detectors positioned on arterial lanes about $100 \mathrm{~m}$ upstream of most major intersections in our area provided 5-min vehicle counts and occupancy measurements.

- Mobile sensors: 140 taxis equipped with GPS and a data logger reported their position and other data with suitable time stamps. Relevant data included activations and deactivations of the parking brake, left blinker and hazard lights, as well as the beginning and end of all stops lasting more than a few seconds. More details are given in Sarvi et al. (2003).

Unfortunately, the taxi data were not linked to a digital map of Yokohama, so it was not possible to tell without a great deal of effort whether or not a taxi had passed over a particular detector at a particular time. Our analysis was constrained by this limitation.

\section{Results from detector data}

We now show that the part of our network covered by detectors has an MFD with less scatter than for individual links, and that the MFD is reproduced under different demand conditions.

\subsection{Existence of the MFD}

Denote by $i$ and $l_{i}$ a road lane segment between intersections and its length; and by $q_{i}$ and $o_{i}$ the flow and occupancy measured by the corresponding detector in a particular time slice. We use $A$ for the set of lane segments in our study area, and $A^{\prime} \subset A$ for the subset with detectors. As is well known, the density at a detector location is $k_{i}=o_{i} / s$, where $s$ is the space-mean effective vehicle length, which is about $s \cong 5.5 \mathrm{~m}$ (Kuwahara, 2007). We are interested in patterns in these variables produced by both individual detectors and the complete collection.

To this end, let us define the following weighted and unweighted averages: $q^{w}=\Sigma_{i} q_{i} l_{i} / \Sigma_{i} l_{i}$ and $q^{u}=\Sigma_{i} q_{i} / \Sigma_{i} 1$ for flow; and $o^{w}=k^{w} s=\Sigma_{i} o_{i} l_{i} / \Sigma_{i} l_{i}$ and $o^{u}=k^{u} s=\Sigma_{i} o_{i} / \Sigma_{i} 1$ for occupancy and density. Note that the numerator of $q^{w}$ is the production. The unweighted averages are space-means in the sense of Edie (1963) for that disjoint part of the network, $A$ " $\subset A$ ', composed of those parts of our lane segments covered by detectors; i.e., the unweighted averages are representative of $A^{\prime \prime}$. The weighted averages would be space-means for $A^{\prime}$ if the detectors happen to be at representative locations within each link. This should happen automatically for flows (i.e., for production) on time slices large compared with a traffic cycle because on this time scale link flows are roughly the same regardless of where they are measured within a link. But obviously, the same is not necessarily true for density or speed.

Let us look at disaggregated lane data first. Fig. 1a is a scatter-plot of $q_{i}$ vs. $o_{i}$, for a whole weekday with time slices $\Delta t=5 \mathrm{~min}$, greater than the signal cycles, for two different detectors. Note the scatter, especially when flows are maximal $\left(o_{i} \cong 0.3\right)$. This disorder corresponds to a single traffic lane. It persists at the link level, after aggregating data for the lanes of a single link. The disorder persists because among other reasons queue lengths and signal phases vary across time slices. But, would it persist if one aggregated data from all the detectors?

To answer this question data were aggregated for two different days: a weekday (12/14/2001) and a weekend day (12/16/2001). Fig. $1 \mathrm{~b}$ and c shows the time-series of average flows and occupancies, $q^{u}$ and $o^{u}$, that were observed. Note how at the time of maximum occupancy (around 17:00 h on both weekdays and week ends) the average flow is sub-maximal on the weekday - but not on the weekend. This indicates severe congestion in the weekday's afternoon rush hour, but not on the weekend. Note as well that flow and occupancy varied considerably by time-of-day, on both days. These substantial variations within and across days suggest that the demand rates and origin-destination $(\mathrm{O}-\mathrm{D})$ tables varied considerably during our observations. 

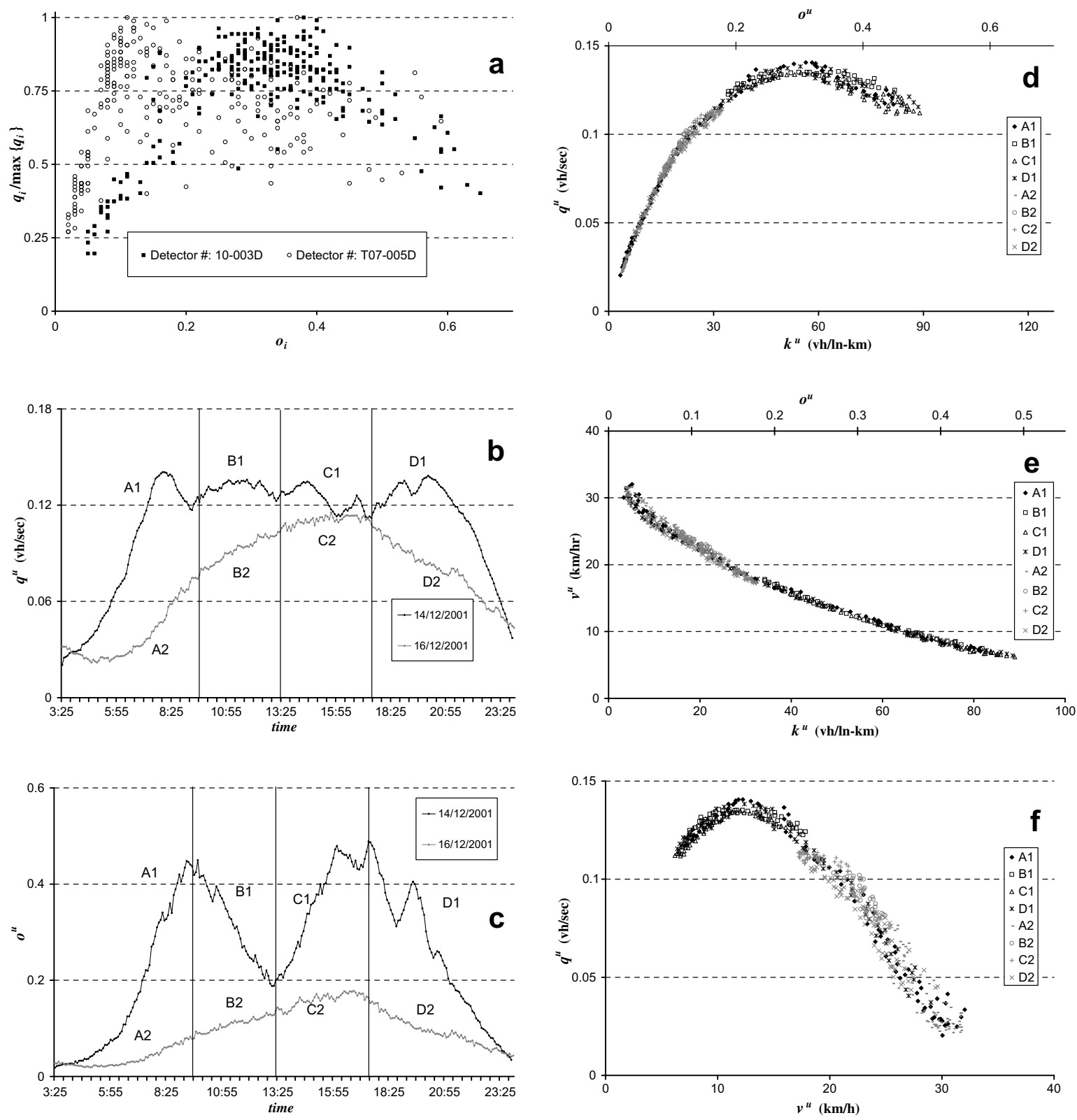

Fig. 1. Loop detector data: (a) flow vs. occupancy pairs for two single detectors across a day; (b) time-series of average flow; (c) time-series of average occupancy; (d) average flow vs. average occupancy from all the detectors across two different days; (e) average speed vs. average occupancy; and (f) average flow vs. average speed.

Fig. 1d,e, and $\mathrm{f}$ is scatter plots of pairs of: $q^{u}, k^{u}=o^{u} / s$ and $v^{u}=q^{u} / k^{u}$. These are the averages representative of the detector locations $A^{\prime \prime} \subset A^{\prime}$. From the high degree of ordering (compare with Fig. 1a) we conclude that an MFD exists on the disjoint portion of the network covered by detectors. Plots involving weighted averages exhibit similar low scatter. Of practical interest are plots involving $q^{w}$, because $q^{w}$ is the production per unit length on all of $A^{\prime}$. Fig. 2 shows how well $q^{w}$ can be predicted from the detector data alone.

The different symbols in Figs. 1d,f and 2 correspond to the 8 time periods of our study shown in Fig. 1b and c. Note how each set of symbols describes an MFD that cannot be distinguished from the others despite the substantial variations in $\mathrm{O}-\mathrm{D}$ demands across time periods. 


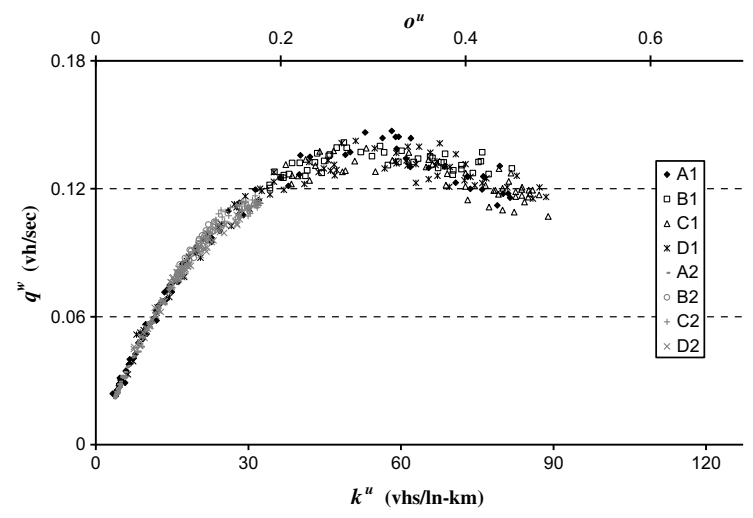

Fig. 2. Weighted average flow vs. unweighted average occupancy.

Note as well (see Fig. 1f) that the flow-speed relation is not monotonic as mentioned in the introduction; that the maximum flow is reached for an average occupancy of 0.3 (Fig. 1d) and an average speed of $13 \mathrm{~km} / \mathrm{h}$ (Fig. 1f); and that these markers are consistent across time periods (morning and evening peaks). Furthermore, the hysteresis phenomenon first reported for individual lanes in Treiterer and Myers (1974) is notable for its absence. ${ }^{2}$ The consistency of these results indicates that if the trip completion rate in $A$ ' is linearly related to $q^{w}$ then the perimeter control rule in Daganzo (2007) would increase the accessibility delivered by $A^{\prime}$, just as predicted in that reference. Given Yokohama's heavy congestion, its residents would benefit substantially from its application. Therefore, we now examine the relationship between the trip completion rate and $q^{w}$.

\subsection{Existence of a linear relation between exit flows and network flow}

From an accessibility standpoint, including the dynamics of the rush hour, we are interested in the connection between the unit production $q^{w}$ and the rate at which cars reach their destinations - defined for now as leaving $A^{\prime}$. Cars can leave $A^{\prime}$ along the perimeter of the region and also internally, but they are most readily observed along the perimeter where there are detectors. Accordingly, we shall focus for now on this observed outbound perimeter flow $(\mathrm{veh} / \mathrm{h})$, which we denote $D^{\prime}$. This perimeter flow includes a considerable part of the trip-ends, as we shall see later. So, a connection between $D^{\prime}$ and $q^{w}$ would shed some light on the general accessibility question, although it cannot be the final answer.

Fig. 3a shows the time series of $D^{\prime}$ and $q^{w}$ for our weekday when the flows are sampled in 5-min intervals. $D^{\prime}$ has been calculated from the detectors located on the periphery of $A$. They appear to be correlated. Other weekdays are similar. Fig. $3 \mathrm{~b}$ confirms this fact; it reveals that the ratio $q^{w} / D^{\prime}$ is close to 0.033 in every 5 -min time slice of the day. ${ }^{3}$

Section 4 below analyzes taxi data to expand the results of this section to $A$; it will also demonstrate that a trip completion relation similar to Fig. $3 \mathrm{~b}$, but including all trips and not just those exiting in the perimeter, also holds for $A$. The findings are important for two reasons: (i) because they establish that the MFD is not a property of the detectors or their locations - only of the network itself and (ii) because perimeter control can be more easily applied to $A$ than $A$ '.

\footnotetext{
${ }^{2}$ Daganzo (2002) attributed the hysteresis phenomenon to lane changing and the non-conservative nature of flow in a single lane. The fact that the hysteresis loop disappears when we aggregate lanes supports this interpretation.

${ }^{3}$ Note there is no trend. In fact, the best-fit 5-degree polynomial deviates from the straight line by less than $0.2 \%$ (RMS); and the difference between the two curves is statistically insignificant. The residuals are completely explained by statistical variations in trip lengths across individual cars.
} 

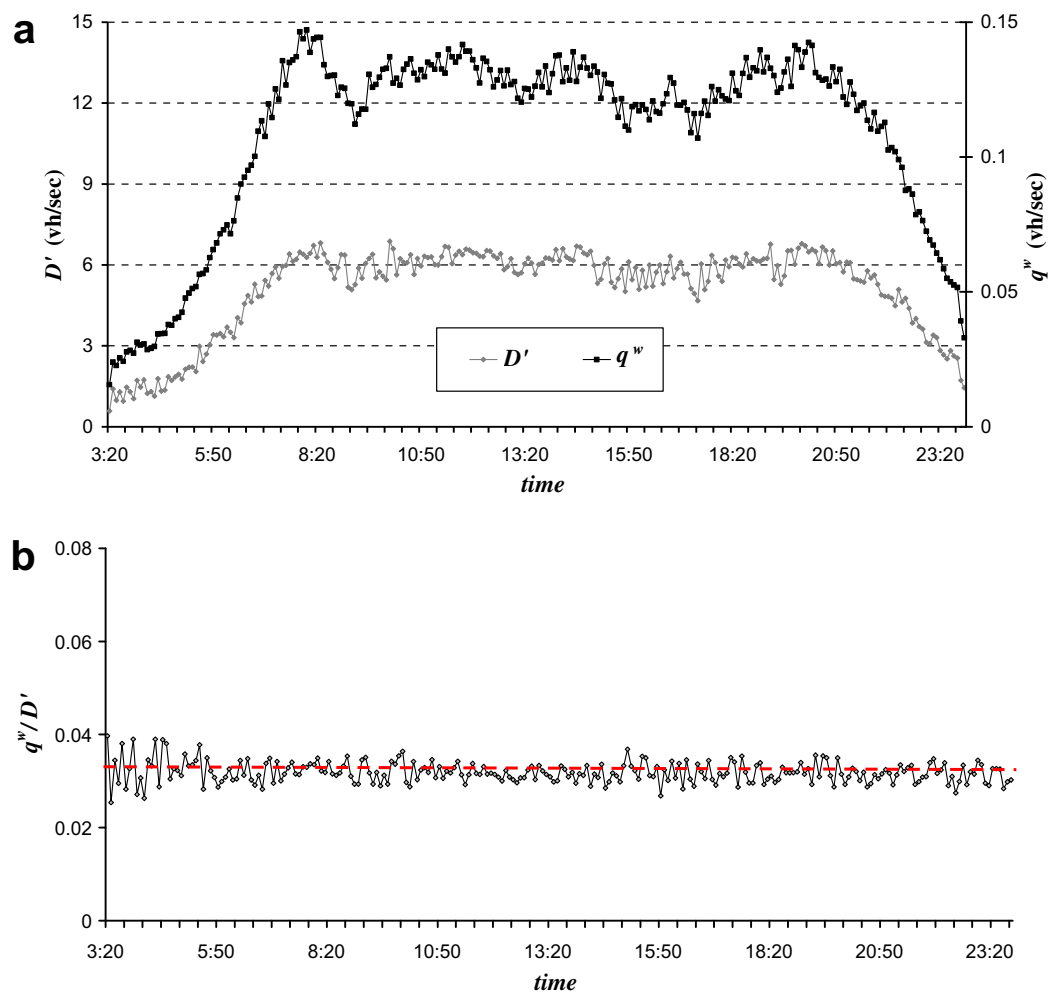

Fig. 3. Average network flow (production per unit length) and exit rates measured by Yokohama's detectors on 12/14/2001 (5-min time slices): (a) time series of the two variables and (b) time series of their ratio.

\section{Results from taxi data}

We assume that taxi passengers follow routes through Yokohama's center similar to those of cars. Then, we can infer the flow of all cars in $A$ by scaling up the observed flow of all cars in $A$ ' with a factor determined from the observed flows of passenger-carrying (full) taxis in $A$ and $A^{\prime}$. With this scaling method we can also infer other features of $A$, such as the trip completion rate, the vehicular accumulation and the space-mean speed. The key ingredient in these estimation recipes is a set of valid (full) taxi trips. [These trips should be roughly representative of car trips with respect to average speed since taxi stops that are not due to congestion are filtered, and also with respect to average distance traveled per trip completion since the distance traveled by a car and a taxi passenger should be similar. ${ }^{4}$ ] Section 4.1, describes how this set was identified and Section 4.2 the estimation results.

It should be reassuring that the full taxi vs. car similarity hypothesis is confirmed by the data set of Section 4.1: Fig. 4 shows the ratios of the outbound vs. inbound flows for full taxis and cars, which vary with time but remain remarkably close to each other throughout the day. ${ }^{5}$

\subsection{Filtering method for passenger-carrying taxis}

Although the events in our data set did not include "boarding" or "alighting" moves, the data set included other information that revealed whether a particular stop (even if it occurred outside $A$ ) was a passenger move in or out of the taxi. From this we identified the valid trips.

\footnotetext{
${ }^{4}$ Car distances could be significantly larger than taxi distances if finding parking requires much extra travel, but this effect is minor in Yokohama.

${ }^{5}$ Significant fluctuations arise but only when the number of full taxis is low $(<10)$, as expected.
} 


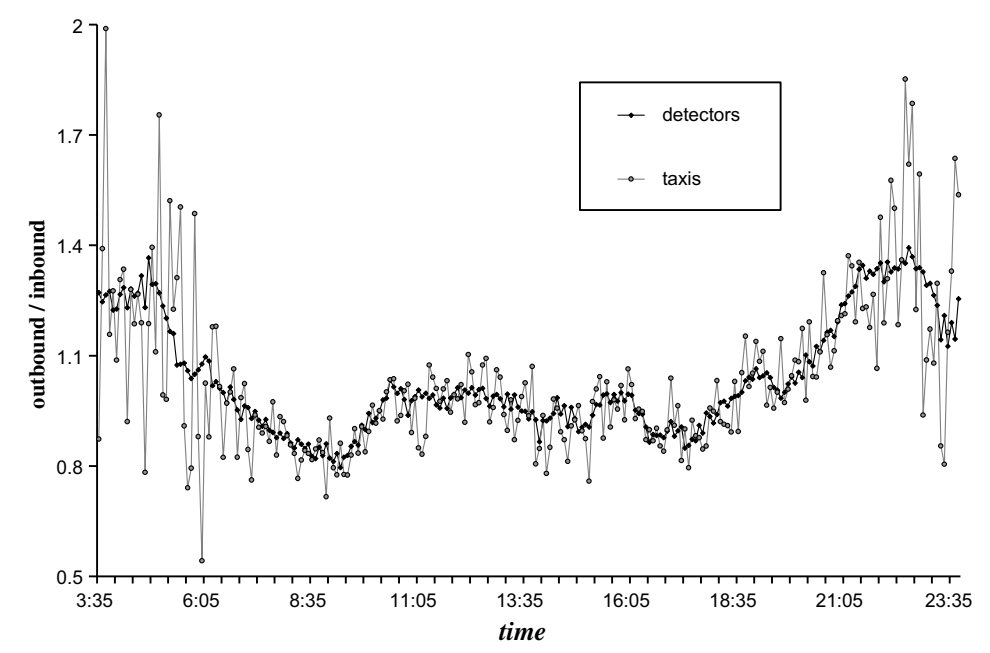

Fig. 4. Observed outbound/inbound flow ratio for all cars (detectors) and full taxis.

To tag a stop as a passenger move, the taxi had to do one or more of the following: (i) turn on the hazard lights; (ii) use the parking brake; (iii) turn on the left turn light and stop for at least $45 \mathrm{~s}^{6}{ }^{6}$ or (iv) continuously exhibit a speed of less than $3 \mathrm{~km} / \mathrm{h}$ for more than $60 \mathrm{~s}$. Condition (iv) was used to capture stops where the taxi driver does not activate any safety devices when serving a passenger, while filtering out stops due to traffic congestion. The $3 \mathrm{~km} / \mathrm{h}$ limit is commensurate with the granularity of our data.

The path traveled by a taxi between two consecutive passenger moves is a trip. A taxi route is an alternation of full and empty trips, and the following two-part criterion was used to identify valid (full) trips: (a) the trip lasts more than $5 \mathrm{~min}$ and is longer than $1.5 \mathrm{~km}$; and (b) the trip distance is less than twice the Euclidean distance between its end points. Condition (a) rules out short trips, which are unlikely to be filled with a passenger, and in any case would not be representative of car trips. Condition (b) disallows circuitous routes, which for the most part taxis employ only while cruising for passengers.

Only on very rare occasions, as happens in reality, our method identified consecutive full trips. This indicates that the method was effective in censoring out spurious stops. We plotted many taxi routes and the patterns looked realistic. Fig. 5 shows by means of white lines the complete set of taxi routes for one week, which accurately reproduce the area's map. The perimeter of $A$ is shown by a dashed line. The figure also shows the trajectory of taxi-1807 for $3 \mathrm{~h}$. The large symbols $\mathrm{A}_{1}$ to $\mathrm{A}_{7}$ depict passenger moves: black for boarding and grey for alighting. These points define an alternating sequence of full and empty trips. Smaller symbols have been used to time-stamp the position of the taxi every $30 \mathrm{~s}$. Note how the distance between consecutive symbols is greater when the taxi is full than when it is not.

\subsection{Existence of an MFD in A: estimation results}

The filtered taxi data were aggregated into 5-min intervals matching those used in Section 3 with the loop detectors, and from this information we calculated for each time slice and for all the full taxis: (i) the total distance $\delta$ traveled in $A$; (ii) the total time $\tau$ spent in $A$; (iii) their space-mean speed in $A, v_{\mathrm{T}}=\delta / \tau$; (iv) their number in $A, n_{\mathrm{T}}=\tau / \Delta t$; (v) the number $N_{\mathrm{T}}$ that exited $A$ along its perimeter; and (vi) the number $M_{\mathrm{T}}$ that finished a valid trip inside $A$. These data were then used to estimate the space-mean speed $v$ and accumulation $n$ of all cars in $A$ to see if, despite the statistical errors due to the low number of taxis, an MFD as in Fig 1e appears.

\footnotetext{
${ }^{6}$ People in Yokohama drive on the left side of the streets.
} 


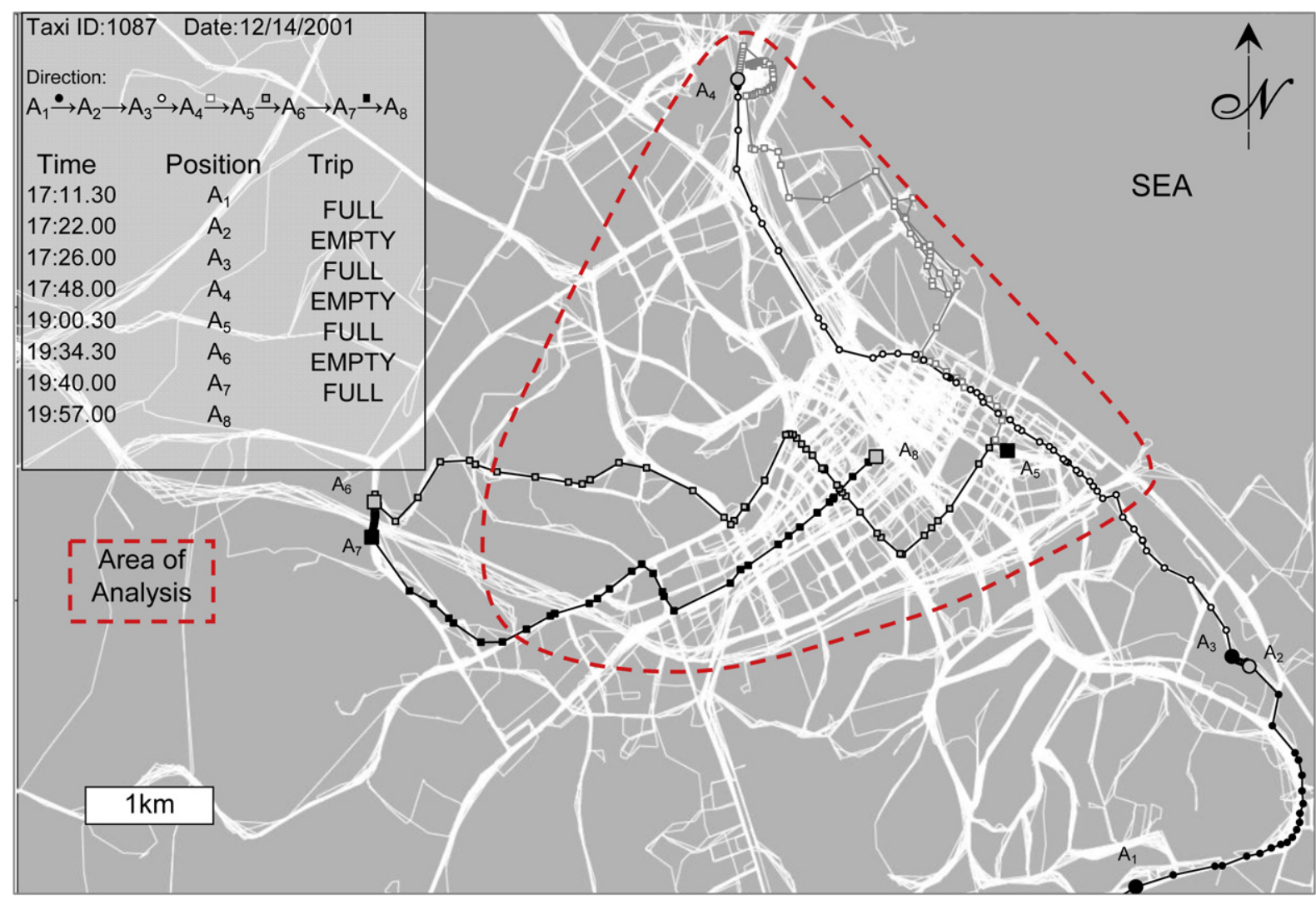

Fig. 5. Trajectory of taxi 1087, and area map (in white) produced by a superposition of all the taxi trajectories.

We use $v_{\mathrm{T}}$ as our estimate $\hat{v}$ for $v$, since valid taxi trips are assumed to be typical trips; i.e.:

$$
\hat{v} \equiv v_{\mathrm{T}} \cong v
$$

However, if $\delta$ and $\tau$ are low (e.g., as occurs at night) the estimate could have a significant error due to insufficient coverage. To alleviate this problem we aggregated our data and used $\Delta t=30 \mathrm{~min}$. This ensures that the coverage of $A$ is reasonable when the number of full taxis in $A$ is on the order of $10-$ a value that is consistently exceeded during the day time hours.

The estimation of density is more complicated. First, since the number of lane-km in $A$ is unknown but fixed we used the accumulation $n=k L$ as a proxy. Thus, an estimate $\hat{n}$ was constructed as follows: Let $N^{\prime}=D^{\prime} \Delta t$ and $N_{\mathrm{T}}^{\prime}$ be the numbers of vehicles and taxis exiting $A^{\prime}$ along streets with detectors. The former is measured by our detectors, and the latter is approximated as a fixed proportion of $N_{\mathrm{T}}$, which is also observed. We used $N^{\prime}{ }_{\mathrm{T}} \cong 0.7 N_{\mathrm{T}}$. Measurements of $N^{\prime}{ }_{\mathrm{T}}$ could not be automatically extracted from the database because the taxi data were not linked to a digital map of Yokohama; i.e. we had no easy way of knowing on which particular street the taxis were exiting. The factor " 0.7 " was estimated manually, after tracing for a whole day the routes of 10 taxis on the map of Fig. 5, and determining from this map whether each exit point belonged either to $A^{\prime}$ or $A-A^{\prime}$. Fig. 6 displays the result of this effort. Note how the fraction of exits that use $A^{\prime}$ varies little from 0.7 . Since full taxis are representative of ordinary vehicles, we expect the ratio $n / N^{\prime}$ to equal on average $n_{\mathrm{T}} / N^{\prime}{ }_{\mathrm{T}}$. Therefore, we can estimate $n$ with $\hat{n}$, as follows:

$$
n \cong n_{\mathrm{T}} N^{\prime} / N^{\prime}{ }_{\mathrm{T}} \cong n_{\mathrm{T}} N^{\prime} /\left[0.7 N_{\mathrm{T}}\right] \equiv p n_{\mathrm{T}} \equiv \hat{n},
$$

where $p \equiv N^{\prime} /\left[0.7 N_{\mathrm{T}}\right]$ is an estimated but observable expansion factor, which approximates the ratio of vehicles vs. full taxis exiting $A^{\prime}$ along streets with detectors. 


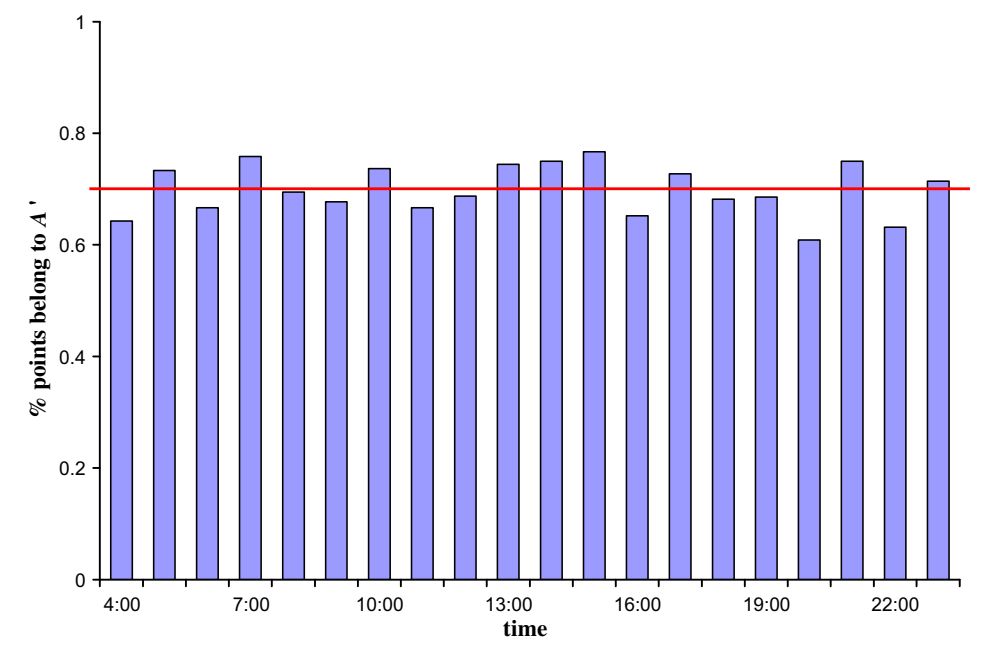

Fig. 6. Fraction of full taxis exiting the perimeter of $A$ that exit through $A$ ' at different times of the day.

Fig. 7a is a scatter plot of $\hat{v}$ vs. $\hat{n}$. Time slices are displayed every $5 \mathrm{~min}$, so there are 12 data points per hour even though $\Delta t=30 \mathrm{~min}$. Triangles are used for the morning and circles for the afternoon and evening. Triangles of consecutive time slices are linked by a dark line. The figure clearly shows that the pattern is the same at all times of the day, without hysteresis, and that the points cluster along an invisible curve; i.e., an MFD exists in $A$.

What about the scatter, though? We find that it can be completely explained by the experimental errors resulting from the low number of taxis. These errors are of three sorts, each corresponding to one of the approximate equalities in (1a) and (1b). The most severe of these is the first approximate equality of (1b). Because taxis leave $A$ randomly (approximately as a Poisson process) we estimate that this error is on average about $N_{\mathrm{T}}^{,-1 / 2}$ when $N_{\mathrm{T}}^{\prime}>25$. Fig. $7 \mathrm{~b}$ shows the 1- and 2-standard deviation bands arising from this formula on each side of a fitted curve. ${ }^{7}$ The bands only apply to those points (shaded circles) with $N^{\prime}{ }_{\mathrm{T}}>25$. The white squares arise mostly during the night and their higher scatter should be expected both because our approximation does not hold when $N^{\prime}{ }_{\mathrm{T}}$ is low, and also because (1a) then introduces significant errors in the vertical direction.

\subsection{Existence of a linear relation between the trip completion rate in $A$ and total production in $A$}

We estimate the trip completion rate $D$ (i.e. the rate at which vehicles depart our network including trips that end within the study area) by $\hat{D}=p\left(N_{\mathrm{T}}+M_{\mathrm{T})} / \Delta t\right.$; i.e., by expanding the rate at which full taxis exit $A$ or finish a trip within $A$. The space-mean network flow $q$ cannot be estimated in the same way because we do not know the total network length $L$ required to express the space-mean flow of full taxis in $A$, which is $\delta / \Delta t L$. Therefore, instead of $q$ we estimate its proxy, the travel production, $P=q L$, and focus on the relation between production and the trip completion rate. Since the full-taxi production in $A$ is $\delta / \Delta t$, we estimate total production in $A$ by $\hat{P}=P \delta / \Delta t$.

Fig. 8 is a time-series of $\hat{P} / \hat{D}$ which supports the hypothesis that $\hat{P} / \hat{D}$ is relatively constant and equal to about $2.3 \mathrm{~km} .{ }^{8}$ This constant is the average vehicular trip length in $A$.

\footnotetext{
${ }^{7}$ We took the curve that best fits Fig. 1d, and scaled it horizontally by the factor that resulted in the best agreement, $L=131$ lane-km. This factor, however, is not necessarily the true length of $A$.

${ }^{8}$ Again a 5-degree polynomial does not improve the fit in a statistically significant way, and the best fitted polynomial has an RMS $<0.1 \%$
} 

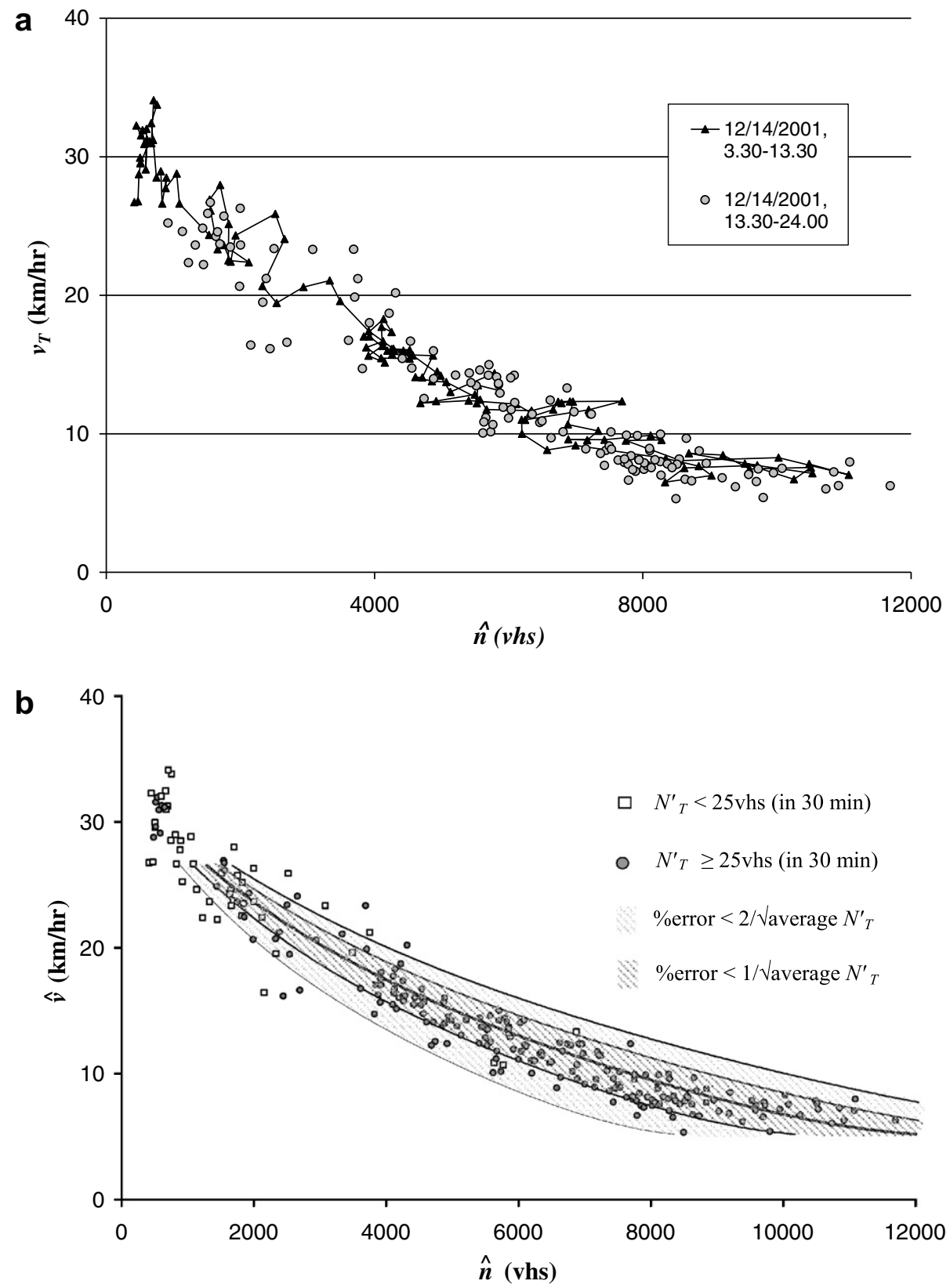

Fig. 7. Yokohama's estimated MFD: (a) Scatter plot of $\hat{v}$ vs. $\hat{n}$ and (b) 1- and 2-standard deviation bands.

\section{Discussion}

The results in this paper show that neighborhoods on the order of $10 \mathrm{~km}^{2}$ in cities like Yokohama, Japan, should have a well-defined MFD. This MFD can be used to improve accessibility as measured by the city's trip completion rate. This can be done with pricing, rationing and/or perimeter control strategies based on neighborhood accumulation and speeds, such as those proposed in Daganzo (2007) and Geroliminis and Daganzo (2007). Simple versions of these strategies are already being used: e.g., in London, Stockholm and Singapore (pricing); in Beijing - a test in anticipation of the 2008-Olympiad - and Mexico City (rationing); and in Zurich (perimeter traffic control). But by knowing the MFD and monitoring the state of traffic continuously, 


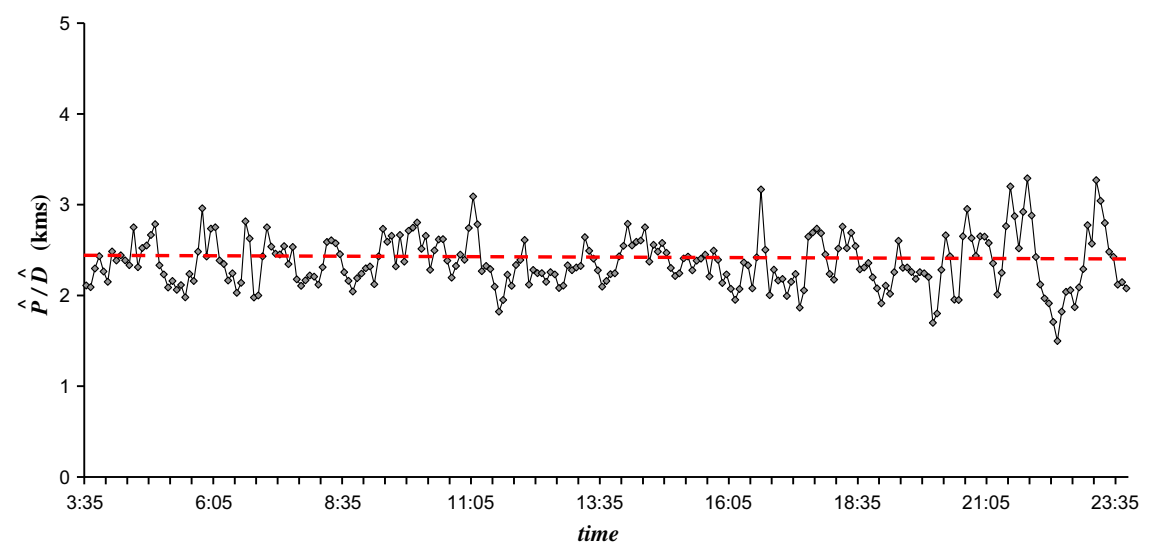

Fig. 8. Total production over trip completion rate time-series $(12 / 14 / 2001)$.

transportation managers can now see whether their system is in a state that is producing the desired accessibility levels for all modes and at all times. Therefore, existing strategies can be refined.

The main caveat in the claims of this paper is that, unlike taxi trips, some automobile trips with internal destinations include a "looking-for-parking" portion that extends their length, and this extension increases accumulation. This effect should be minor in our study because $70 \%$ of the trips were found to have external destinations and, surely, a significant portion of internal trips have pre-assigned parking. The looking-forparking phenomenon is important from a policy standpoint, however, and is currently being studied.

Also important from a practical standpoint are the methods used to estimate the state of a neighborhood's network (its space-mean speed and/or accumulation) which trigger the control policies along its perimeter. Although many crowded cities that could benefit from these controls do not have a supporting infrastructure of fixed detectors to monitor their state, these cities often have vehicles equipped with GPS that, like the Yokohama taxis, can serve as city-wide probes. More experimental research is needed to better understand the quantity and character of the probes necessary for a sufficiently accurate estimation of a neighborhood's traffic state. A development effort is also needed to produce middleware that will support these types of control strategies on traffic signals of different types. An effort is underway (Sengupta et al., 2007).

In summary, the results of this paper show that an MFD exists on neighborhood-sized sections of cities independently of the demand; and that it can be used to control demand to improve accessibility. But accessibility can also be improved by modifying the infrastructure, since the amount of street space allocated to cars and buses, street closures, flyover construction or new signal timings surely affect a neighborhood's MFD. Therefore, we are currently studying how a city's MFD depends on its infrastructure.

\section{Acknowledgements}

We thank Professor Masao Kuwahara from the University of Tokyo for providing us the data. This research was supported by the University of California, Berkeley's Center for Future Urban Transport (a Volvo International Center of Excellence).

\section{References}

Daganzo, C.F., 2005. Improving city mobility through gridlock control: an approach and some ideas. U.C. Berkeley Center for Future Urban Transport Working Paper UCB-ITS-VWP-2005-1.

Daganzo, C.F., 2007. Urban gridlock: macroscopic modeling and mitigation approaches. Transportation Research Part B 41 (1), $49-62$.

Daganzo, C.F., 2002. A behavioral theory of multi-lane traffic flow part I: long homogeneous freeway sections. Transportation Research Part B 36 (2), 131-158.

Edie, L.C., 1963. Discussion of traffic stream measurements and definitions. In: Almond, J. (Ed.). Proceedings of the 2nd International Symposium on the Theory of Traffic Flow. OECD, Paris, France, pp. 139-154. 
Geroliminis, N., Daganzo, C.F., 2007. Macroscopic modeling of traffic in cities. In: TRB 86th Annual Meeting, \# 07-0413, Washington, DC.

Herman, R., Ardekani, S.A., 1984. Characterizing traffic conditions in urban areas. Transportation Science 18 (2), $101-140$.

Herman, R., Prigogine, I., 1979. A two-fluid approach to town traffic. Science 204, 148-151.

Kuwahara, M., 2007. Private Communication.

Sarvi, M., Horiguchi, R., Kuwahara, M., Shimizu, Y., Sato A., Sugisaki Y., 2003. A methodology to identify traffic condition using intelligent probe vehicles. In: Proceedings of the 10th ITS World Congress, Madrid.

Sengupta, R ., Manasseh, C., Levenson, S., 2007. Service oriented architectures for VII - extending web services to the roadway. In: ITS America's 2007 Annual Meeting and Exposition, Palm Springs, CA.

Smeed, R.J., 1966. Road capacity of city centers. Traffic Engineering and Control 8 (7), 455-458.

Thomson, J.M., 1967. Speeds and flows of traffic in Central London: 2. Speed-flow relations. Traffic Engineering and Control 8 (12), $721-$ 725.

Treiterer, J., Myers, J.A., 1974. The hysteresis phenomenon in traffic flow. In: Buckley, D.J. (Ed.), Proceedings of the 6th International Symposium on Transportation and Traffic Theory, pp. 13-38.

Wardrop, J.G., 1968. Journey speed and flow in central urban areas. Traffic Engineering and Control 9 (11), 528-532.

Zahavi, Y., 1972. Traffic performance evaluation of road networks by the $\alpha$-relationship. Parts I and II. Traffic Engineering and Control 14 (5 and 6), 228-231, 292-293. 